Wilson, A. T. M. (1939). Brit. F. med. Psychol. r8, I12.

Wolf, S. (1949). Ann. intern. Med, 31, 637.

Wolf, S. \& Glass, G. B. J. (1950). Proc. Ass. Res. nerv. Dis. 29, 665.

Wolf, S. \& Wolff, H. G. (1943). Human Gastric Function. Oxford: University Press.

\title{
Anorexia Nervosa
}

\section{By C. F. Rolland, Department of Therapeutics, The Royal Infirmary, Edinburgh}

Anorexia nervosa may be looked upon, in a sense, as the antithesis of obesity in that it is a disorder of appetite leading to a reduced intake of food and a resultant fall in body-weight. It differs from obesity in two other important aspects. First, it is relatively uncommon; for example only twenty-five cases have been treated in Professor D. M. Dunlop's wards in the Royal Infirmary of Edinburgh in the past I 5 years. Secondly, the condition is brought about by the development of a serious 'morbid mental state', as was recognized by Sir William Gull (I874) in his classical description of the disease, though modern work suggests that obesity may have certain psychological connotations, it can hardly be said to arise from serious mental disorder.

\section{Aetiology}

It is not difficult to recognize the significance of the 'morbid mental state' in the aetiology of anorexia nervosa, but it is much less easy to define the nature of the mental disturbance leading to such a marked abnormality of behaviourthe self-starvation, which is the most striking feature of the disease.

At one time it was popular to describe the patients as sufferers from hysterical anorexia, but there are objections to this classification. Nemiah (I950), who made a detailed analysis of fourteen cases, concluded that the disease was neither an hysterical disorder nor an obsessional state and that it was not yet possible to elaborate a detailed hypothesis about its aetiology. At the same time he emphasized certain features that he considered of importance in the previous histories of his patients. Amongst these he mentioned the frequent occurrence of an overprotective parent, conflicts in the patient's mind between the need to depend upon a parent and the desire for independence, difficulties in forming relationships with other people, an emotional frigidity and problems connected with the sexual significance of the mouth. Ryle ( 1936 ) noticed that the disease was more common in private than in hospital practice and concluded that it occurred in homes 'where sensitive natures and solicitude flourished side by side'. He mentioned several circumstances considered to be initiating factors, such as misguided attempts as 'slimming' and unfortunate love affairs. He was careful to state that there was usually a multiplicity of factors in the aetiology of any individual case. It seems, then, that the disease occurs only when suitable initiating factors operate in the life of one who is susceptible by virtue of her inherited constitution and having experienced certain difficulties in her earlier days. We know something of the nature 
of the difficulties and the initiating factors, but it is often impossible to trace with accuracy the sequence or the full significance of the events that lead to the onset of the disorder in an individual case.

Last year we saw a young patient with an interesting history, which was obtained only with the assistance of an intelligent parent. The patient was a girl of $\mathrm{I} 6$ years who had two brothers, the younger being i I years older than herself. She was very much attached to her elder brother, who spoiled her, and she was rather hostile to her mother who, though unwittingly, frightened her. When she was 13 years old, a girl in her class at school became pregnant. After the child was born the other girls in the class, who had naturally been much intrigued by the experience of their classmate, noticed that the patient was plump and taunted her with suggestions that she was about to become the next young mother. This terrified the patient, whose knowledge of the physiology of reproduction was wholly inadequate, and she determined to reduce her weight in order to emphasize to her classmates, and probably to herself, that she was not pregnant. This she accomplished by deliberate starvation. Later, when she wanted to eat more and was much encouraged to do so by her family, she found that her appetite had failed and she could not eat. By the age of 16 she was emaciated and had further difficulties at home and at work, which may well have played a part in her trouble. In this case the story was less involved and more readily obtained than it usually is, and the patient made rapid and satisfactory improvement in hospital with simple encouragement and suggestion.

In contrast to the ready acceptance of the mental origin of the disease, there is much argument as to the means by which the mental disturbance influences the patient and there are now two broad schools of thought. In the one it is held that the cerebral cortex exerts an influence on the hypothalamus, giving rise to anorexia, and that the resultant starvation is responsible not only for the emaciation but also for a suppression of anterior-pituitary function such as is found in severe malnutrition due to famine. In the other school it is accepted that the hypothalamus is influenced by the cerebral cortex, but it is believed that the anterior pituitary is inhibited directly by the hypothalamus and that the signs of endocrine deficiency, such as amenorrhoea, are brought about in this way rather than by the malnutrition alone. At present there is insufficient evidence to permit a final decision between the two hypotheses, though the former seems the more acceptable. It is of great interest to see how recent experimental work on the hypothalamus (Kennedy, I953) lends some support to these views.

\section{Clinical features}

The patient is usually a young unmarried woman, though there are rare cases in the male sex, and the disease occasionally occurs in older women. The patient seldom complains of her anorexia, though her relations often do, and it is amenorrhoea that commonly leads the patient to consult her doctor. In this way many cases come first to the gynaecologists. Constipation is common and so are regurgitation of food and vague abdominal pains. Emaciation, which is often very severe, is 
the most striking clinical feature, and it is frequently noted that the patients are surprisingly active compared with those who are emaciated for some other reason. Sometimes the breasts are remarkably well preserved, in contrast to the extreme loss of fat from other parts of the body. There is a profuse growth of lanugo-like hair and there is a moderate loss of axillary and pubic hair. The pulse is slow, the blood pressure low and the peripheral circulation poor, with the extremities often cyanosed and cold.

Signs of specific food deficiency are very rare, but we have recently seen a case in which there was severe osteomalacia and oedema of the legs. Tests of endocrine function show evidence of a moderate hypopituitarism in the more advanced cases. There is a low metabolic rate, the 17 -ketosteroid excretion is reduced and there is an increased sensitivity to insulin. A single injection of ACTH leads to only a moderate fall in circulating eosinophils. Metabolic studies are difficult to perform in these patients, because they do not co-operate well, and it is impossible to be certain of their food intake unless they are under constant supervision. Such observations as we have been able to make suggest that they behave very like the sufferer from famine. For example, they maintain nitrogen equilibrium even on a very low intake of protein.

\section{Differential diagnosis}

The differential diagnosis of anorexia nervosa is largely concerned with its distinction from organic hypopituitarism or Simmond's disease. This is mainly because the early description of Simmond's disease placed undue emphasis on the occurrence of cachexia in that condition, with the result that it was the wrong diagnosis of many cases of anorexia nervosa. It then became a fashionable examination exercise to distinguish the two diseases by reference to the results of a series of tests of endocrine function, which showed gross impairment in Simmond's disease and less gross in anorexia nervosa. It is now realized that the diagnosis can usually be made on clinical grounds alone. The young, relatively active and grossly emaciated woman with anorexia nervosa is readily contrasted with the older, inactive patient with Simmond's disease, who has a history of a severe postpartum haemorrhage or obvious signs of organic pituitary damage and is seldom emaciated except in the terminal stages of the disease. The introduction of ACTH adds a therapeutic test to our means of differentiation, for it causes dramatic improvement in Simmond's disease but is very disappointing in anorexia nervosa. It goes without saying that it is important to eliminate the possibility of other diseases that may cause severe wasting, such as hyperthyroidism, diabetes mellitus, tuberculosis, malignant disease and drug addiction, before making a diagnosis of anorexia nervosa.

\section{Prognosis}

There is a mortality rate of between 5 and $10 \%$ due to starvation and intercurrent infections. The majority of patients improve to a considerable extent. Many achieve a normal weight and some even fly to the opposite extreme and 
become obese. In this connexion it is of interest that Nemiah (1950) observed episodes of hyperphagia in some of his patients even when they were emaciated. Even though they gain weight many patients remain maladjusted in their mental health. Amenorrhoea is usually the last symptom to disappear and may continue long after the patient is otherwise well.

\section{Treatment}

The treatment of anorexia nervosa depends in the first place on sensible general management and particularly on skilled nursing. The assistance of a wise practical psychiatrist often makes all the difference between success and failure. It is usually advisable to remove the patient from her home, but the choice between a private room, a hospital ward or a mental hospital may be difficult, and it is sometimes necessary to change one's initial plan. One has to try to persuade the patient to eat by every art and device of explanation, encouragement and entreaty, and one has to watch carefully to avoid deceit. We have had patients who have vomited at each visit to the lavatory, who have dosed themselves with cathartics, who have posted food home with dirty clothes and even one who cut a hole in the mattress and secreted food in it. The diet should be designed so that each mouthful contains the maximum amount of calories, and it should not be arranged to suit the patient's desires, for these are often bizarre. It is sometimes possible to get the patient to take calcium caseinate suspensions, suitably coloured and flavoured, in the guise of medicines for the treatment of such symptoms as constipation.

In addition to these important general measures, many attempts have been made to stimulate the patient's appetite with drugs. Insulin and testosterone have long been used for this purpose with little or no success. ACTH and cortisone have succeeded them in popularity, but with no more real value. We have treated four patients with ACTH and found that it is easy to raise the body-weight, but only by causing a retention of salt and water. The weight falls again if the treatment is stopped. There is no real increase in appetite. Still more recently isonicotinic-acid hydrazide has been used, but it too seems to be of little or no value as an appetizer in anorexia nervosa.

Finally, despite the suggestions of some workers, psychiatric treatment can be of real value in this disorder and we have repeatedly seen cases in whom no improvement took place until psychotherapy was instituted.

\section{REFERENCES}

Gull, W. (1874). Trans. clin. Soc. Lond. 7, 22 (quoted by Ryle, 1936).

Kennedy, G. C. (1953). Proc. Nutr. Soc. 12, 160.

Nemiah, J. C. (1950). Medicine, Baltimore, 29, 225.

Ryle, J. A. (1936). Lancet, 231, 893 . 